\title{
Standardized measurement of auricle: A method of high-precision and reliability based on 3D scanning and Mimics software
}

\author{
DI WANG, HAIYUE JIANG, BO PAN, QINGHUA YANG, LEREN HE, HENGYUN SUN, XIAOBO YU and LIN LIN
}

The Seventh Department of Plastic Surgery, Plastic Surgery Hospital, Chinese Academy of Medical Sciences and Peking Union Medical College, Beijing 100144, P.R. China

Received July 25, 2018; Accepted August 30, 2019

DOI: $10.3892 /$ etm.2019.8159

\begin{abstract}
Objective measurement is important for diagnosing congenital or acquired auricular abnormalities and the evaluation of therapeutic efficacy. However, methods applied in the past were mostly inaccurate and unreliable. The present study aimed to introduce five standardized indices for auricle measurement and present a highly precise and reliable methodology combining three-dimensional (3D) scanning techniques and the Materialise Mimics software for the evaluation of auricle sizes. A total of 20 normal ears were measured independently by four surgeons using the standardized digital method with 3D scanning technique and the traditional manual method. Parameters of the auricle, including the length and width, arc length, cranioauricular height and angle were measured using the Mimics software. Paired t-test, Wilcoxon signed rank test and intra-class correlation coefficients (ICC) were performed on the data to assess the precision, uniformity and observer independence of the method. Pearson's product moment correlation was calculated to assess the correlation between auricle length and width in addition to the correlation between cranioauricular height and angle. No significant differences were indicated between measurements of five auricular parameters made by two surgeons using the digital method. However, significant differences were found using the manual method $(\mathrm{P}<0.01)$. ICC values derived from digital measurements ranged from 0.901 to 0.987 , whereas those derived from manual measurements ranged from 0.526 to 0.807 . These results suggested that the standardized digital method was replicable and reliable compared with the traditional manual method. Pearson's coefficient analysis showed that there was a significant correlation
\end{abstract}

Correspondence to: Professor Lin Lin, The Seventh Department of Plastic Surgery, Plastic Surgery Hospital, Chinese Academy of Medical Sciences and Peking Union Medical College, 33 Badachu Road, Beijing 100144, P.R. China

E-mail: linlin_psh@sina.com

Key words: auricular abnormalities, digital measurement, three-dimensional between cranioauricular height and angle $(\mathrm{P}<0.05)$, but no correlations were found between the height and width of the auricle $(\mathrm{P}>0.05)$. Taken together, data from the present study suggested that measurements of the length and width, arch length, and cranioauricular height and angle of auricles using the standardized digital method combining 3D scanning with the Mimics software were comprehensive, precise, convenient, repeatable and reliable.

\section{Introduction}

The ear is one of the most pivotal sensory organs on the face, such that congenital or acquired auricular abnormalities have negative physical and mental impacts on the patients. Most of these abnormalities, including microtia, anotia, cryptotia, prominent ear and constricted ear, can be diagnosed by physical observation. Surgical and non-surgical methods are selectively used for different conditions $(1,2)$. Although scales of satisfaction and other subjective methods are important for the evaluation of therapeutic efficacy (3), anthropometric methods, designed to objectively appraise the severity of abnormalities and outcomes of therapy, are also indispensable (4).

The length and width of the auricle, cranioauricular height, and cranioauricular angle are most frequently applied in the evaluation of the ear $(5,6)$. The arc length of the auricle is also a suitable index for assessing the size of the auricle but has not been fully determined previously. Among the previous studies, manual measurements of straight-line distances using rulers and calipers were applied most frequently $(5,7,8)$. However, this method is inaccurate and unreliable. Although there have been some attempts at measuring auricular angles, convenient and accurate approaches to address the accuracy of this measurement remain elusive $(8,9)$.

Three-dimensional (3D) digital techniques, which can be used to build models of high similarity, have been developing rapidly in the past two decades (10-12). In the present study, a standardized measurement procedure combining Mimics software and a 3D digital device was used to evaluate five indices for auricle measurements in 20 normal ears. Emphasis was placed on the precision and reliability of this method. 


\section{Materials and methods}

Patients. Ethical approval of the study was obtained from the Institutional Review Board of Plastic Surgery Hospital of Peking Union Medical College. Between August 2017 and January 2018, 20 patients (sex, 12 males and 8 females; age range, 5-15 years; mean age, $7.9 \pm 2.88$ years) with unilateral auricular abnormality at the Department of the First Center of Auricular Reconstruction, Plastic Surgery Hospital, Chinese Academy of Medical Sciences, Peking Union Medical College who were subjected to surgery were randomly selected and enrolled into the study (Beijing, China). All parents or guardians of the patients granted informed consent to participate in the study. Eight right normal ears and 12 left normal ears were scanned and measured prior to surgery.

3D equipment. A surface 3D scanner (Artec Space Spider; Artec 3D) with a stated resolution of $0.1 \mathrm{~mm}$ and a point accuracy of $\leq 0.03 \mathrm{~mm}$ was used to capture 3D images of the normal auricles. The acquired preliminary data were processed using the Artec Studio software (version 9.0; Artec 3D) by following the surface scan workflow (including rough serial registration, fine registration, global registration, manual alignment and fast fusion). After preliminary treatment, data were exported as stereolithography format files to the Geomagic Studio 2012 software (3D Systems Corporation) for further processing. Using this software, spike removal, filling of holes matching the curvature of the surrounding mesh and minimization of crease angles between the polygons were achieved to obtain accurate 3D models displaying details and sizes of the ears. Following the preparation of digital images, data were imported into the Materialise Mimics software (version 20.0; Materialise NV) for precise measurements.

Measurement of auricular parameters. The auricular parameters measured included the length of the auricle (LA), the width of the auricle (WA), arch length of the auricle (ALA), cranioauricular height $(\mathrm{CH})$ and cranioauricular angle (CA). LA, WA, ALA, CH, and CA were calculated in the measurement model of the Mimics software. The definitions for these five parameters were as follows: LA, the maximum distance from the sup-helix to the sub-lobe (a-b; Fig. 1A); WA, the maximum distance from the pre-auricle to the post-auricle (c-d; Fig. 1A); ALA, the arc length between the intersection of crus of helix and the tragus and the intersection of earlobe and tragus (e-f; Fig. 1B); $\mathrm{CH}$, the projection of the helical rim at the horizontal plane of the prominent point of tragus to the mastoid ( $\mathrm{g}-\mathrm{h}$; Fig. $1 \mathrm{C}$ and $\mathrm{E}$ ); and $\mathrm{CA}$, the angle formed between the plane of auricle and the plane of mastoid at the horizontal level of the prominent point of tragus (g-h; Fig. 1D and F).

Surgeons 1 and 2 independently measured all 20 normal ears using the digital measurement method with an Artec Spider Device and the measurement points designated on the software, whilst surgeons 3 and 4 independently measured all 20 normal ears using a flexible tape and protractor, without using the standardized indices. Measurement points were chosen subjectively by surgeons 3 and 4 according to prior experience.

Statistical analysis. Each patient was measured three times by each surgeon and the mean value was calculated. All data were imported into Statistical Package for Social Sciences (SPSS) software (version 20.0; IBM Corp.) and analyzed. Distribution of data was analyzed using the Shapiro-Wilk normality test $(\mathrm{P}>0.05)$. Paired samples t-test and Wilcoxon signed rank test were used and $\mathrm{P}<0.05$ was considered to indicate a statistically significant difference. Five sets of data were analyzed using intra-class correlation coefficients (ICC). The level of clinical significance is considered poor, fair, good and excellent when ICC $<0.40,0.40<$ ICC $<0.59,0.60<$ ICC $<0.74$, and $0.75<\mathrm{ICC}<1.00$, respectively (13). Pearson product moment correlation was calculated to reflect the correlation between LA and WA, and between $\mathrm{CH}$ and CA.

\section{Results}

Summary of data. Age, sex, measured side and measured data of the five parameters of all 20 patients are listed in Tables I and II. The mean value of LA measured by surgeon 1 to 4 was $8.64 \pm 4.07,58.59 \pm 3.95,60.02 \pm 4.30$ and $56.83 \pm 3.65 \mathrm{~mm}$, respectively, while that of WA was $31.80 \pm 2.92,31.82 \pm 2.71$, $32.51 \pm 2.69$, and $31.41 \pm 3.01 \mathrm{~mm}$. The mean value of LA was $135.70 \pm 6.56,135.57 \pm 7.13,139.69 \pm 7.27$ and $134.56 \pm 7.48 \mathrm{~mm}$, respectively. The mean value of $\mathrm{CH}$ was $19.43 \pm 2.51$, $19.67 \pm 2.62,18.35 \pm 2.02$ and $20.32 \pm 2.78 \mathrm{~mm}$, respectively. The mean value of $\mathrm{CA}$ was $85.91 \pm 14.54^{\circ}, 86.55 \pm 15.18^{\circ}, 90.6 \pm 3.43^{\circ}$ and $81.8 \pm 13.86^{\circ}$, respectively.

The reliability of ratings or measurements. The $\mathrm{P}$-values and ICC of the five sets of data from two different measurement methods were presented in Tables III and IV. No significant differences were observed in all five sets of measured parameters using the digital method between different investigators ( $P>0.05$; Table III). However, significant differences were observed in the same sets of measured data using the manual method between different investigators $(\mathrm{P}<0.01$; Table IV). ICCs between the two surgeons for LA, WA, ALA, CH and CA measured using standardized methods were $0.98,0.962$, $0.944,0.901$ and 0.987 , respectively (Table III), indicating high precision and observer independence. By contrast, ICC of LA, WA and $\mathrm{CH}$ were calculated to be $0.630,0.526$ and 0.546 , respectively (ICC $<0.7$; Table IV), and 0.790 and 0.807 for ALA and CA, respectively $(0.75<\mathrm{ICC}<0.9$; Table IV) for measurements obtained using the manual method. These results indicated that manual measurements exhibited a lower clinical significance compared with digital measurements.

Linear association between two indices. Pearson product-moment correlation analysis $(\mathrm{r}=0.479 ; \mathrm{P}<0.05)$ showed that a moderate but significant positive correlation was observed between $\mathrm{CH}$ and $\mathrm{CA}$, whilst the same analysis $(r=0.206 ; P>0.05)$ did not demonstrate a significant correlation between LA and WA (Fig. 2).

\section{Discussion}

The present study reported a standardized method combining 3D scanning technique and Mimics software for the evaluation of auricular size. Measurement of auricular size is one of the key elements in the diagnosis, pre-operation design and therapeutic effect assessment of ear abnormalities (14). However, 
A

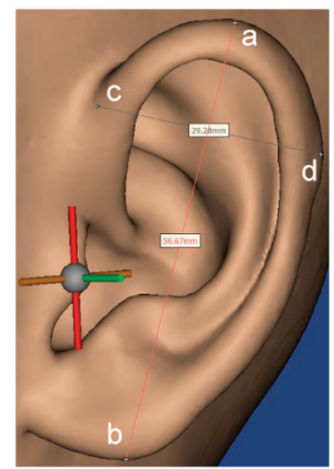

B

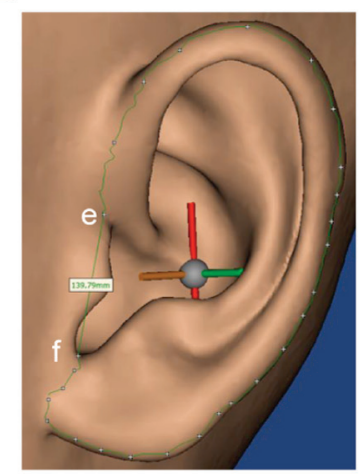

C

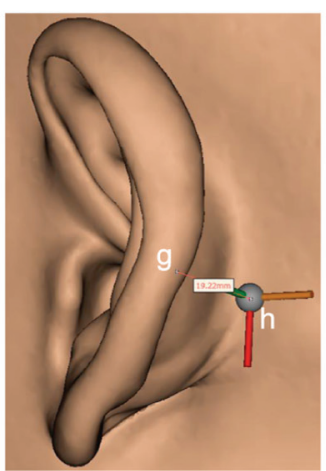

D

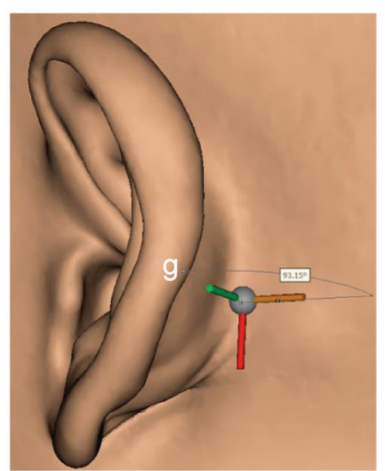

E

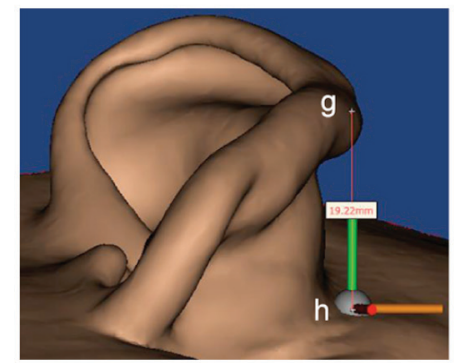

$\mathrm{F}$

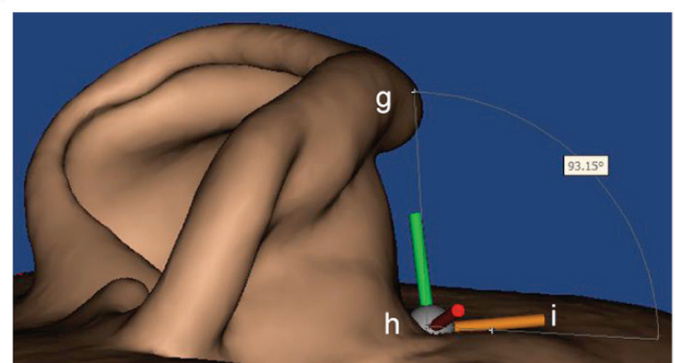

Figure 1. Measurement of length and width of the auricle, arch length of the auricle and cranioauricular height and angle from Mimics software. (A) Anterior view of the auricle indices used for the measurement of the length of the auricle, which is the maximum distance from the sup-helix ' $a$ ' to the sub-lobe 'b', and the width of the auricle, which is the maximum distance from the pre-auricle 'c' to the post-auricle 'd'. (B) Anterior view of the auricle and indices used for the measurement of the arch length of the auricle, which is the arc length between the intersection of crus of helix and tragus ' $e$ ' and the intersection of earlobe and tragus ' $\mathrm{f}$ '. (C) Lateral view of the auricle and indices used for the measurement of cranioauricular height, which is the projection of the helical rim at the horizontal plane of the prominent point of tragus ' $g$ ' to the mastoid ' $h$ '. (D) Lateral view of the cranioauricular angle measurement. (E) Low angle view of the auricle with the indices used for the measurement of cranioauricular height. (F) Low angle view of the indices used for the measurement of the cranioauricular angle (angle ghi), which is the angle between the plane of the auricle and the plane of the mastoid at the horizontal level of the prominent point of tragus. a, the sup-helix; b, the sub-lobe; c, the pre-auricle; d, the post-auricle; e, the intersection of crus of helix and tragus; f, the intersection of earlobe and tragus; $g$, the prominent point of tragus; $h$, the projection of the point $\mathrm{p}$ at the horizontal plane to the mastoid; i, a random point at the horizontal plane across the point $h$.
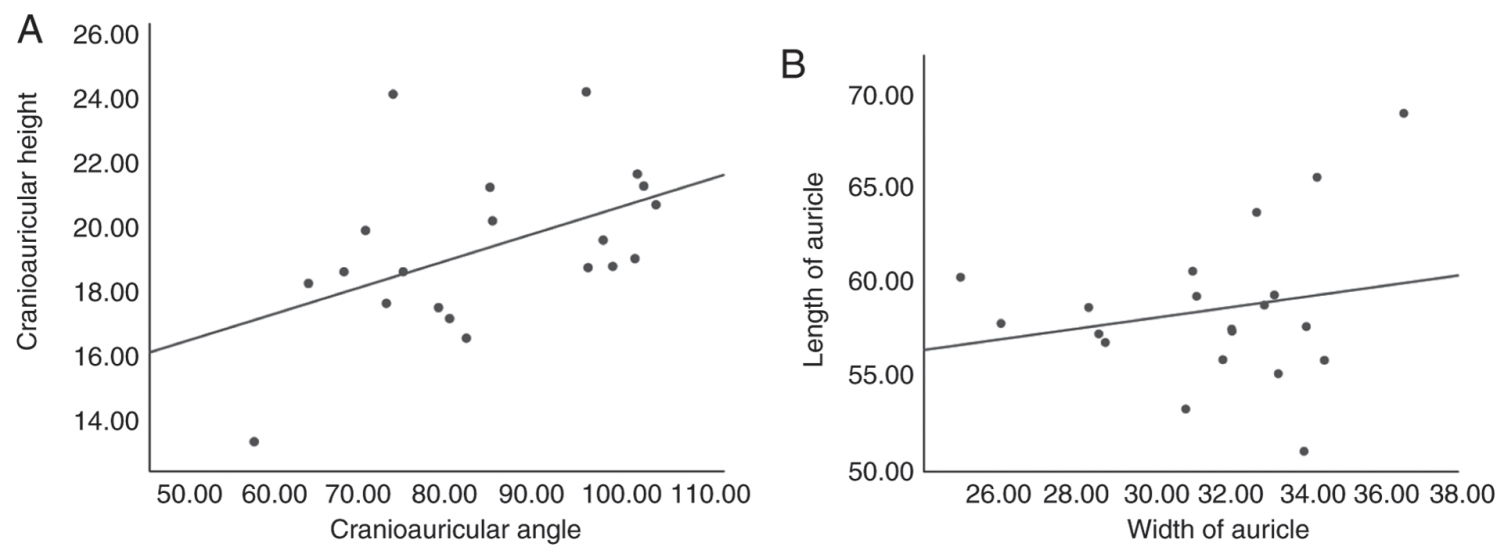

Figure 2. Scatter diagram of Pearson product moment correlation. (A) Correlation between cranioauricular height and angle. (B) Correlation between the length and width of the auricle.

uniform and standardized objective methodology is currently unavailable $(7,9,14)$ and variations exist in the measurement results obtained by different investigators. These differences could lead to errors in surgical or non-surgical therapy for the correction of auricular abnormalities, especially in multiple stage surgery. In previous studies, manual measurement was utilized in the pre- and post-therapy appraisal of auricular abnormalities, where low accuracy and high error rates were reported $(5,15)$. In particular, in the measurement of cranioauricular angle, there are a number of reliable methods, including alginate mold (7). In a previous study, visual inspection was chosen for ear similarity, leading to imprecise outcomes (16). Although innovative methodologies providing auricular measurements have been previously proposed, this 


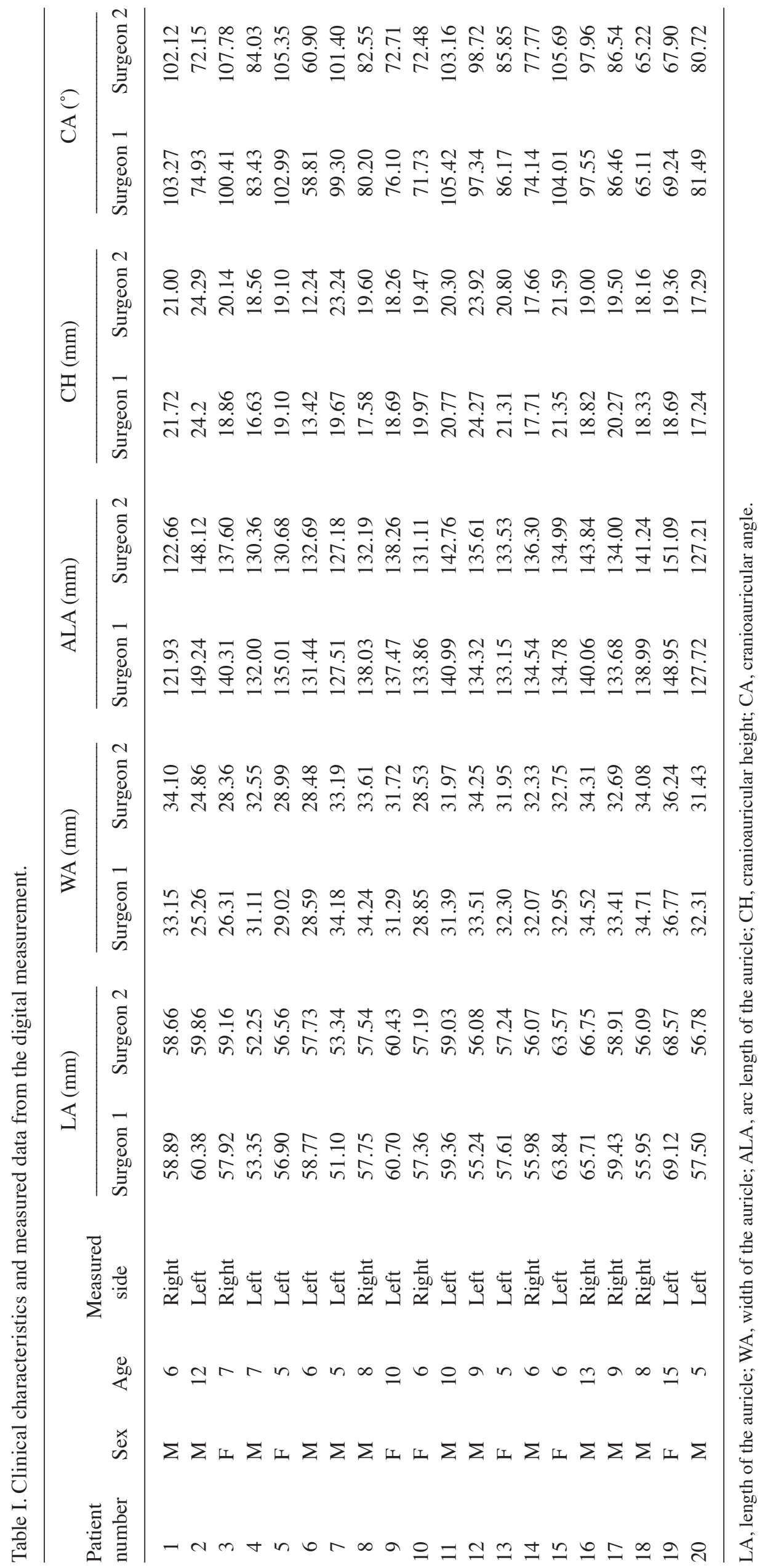




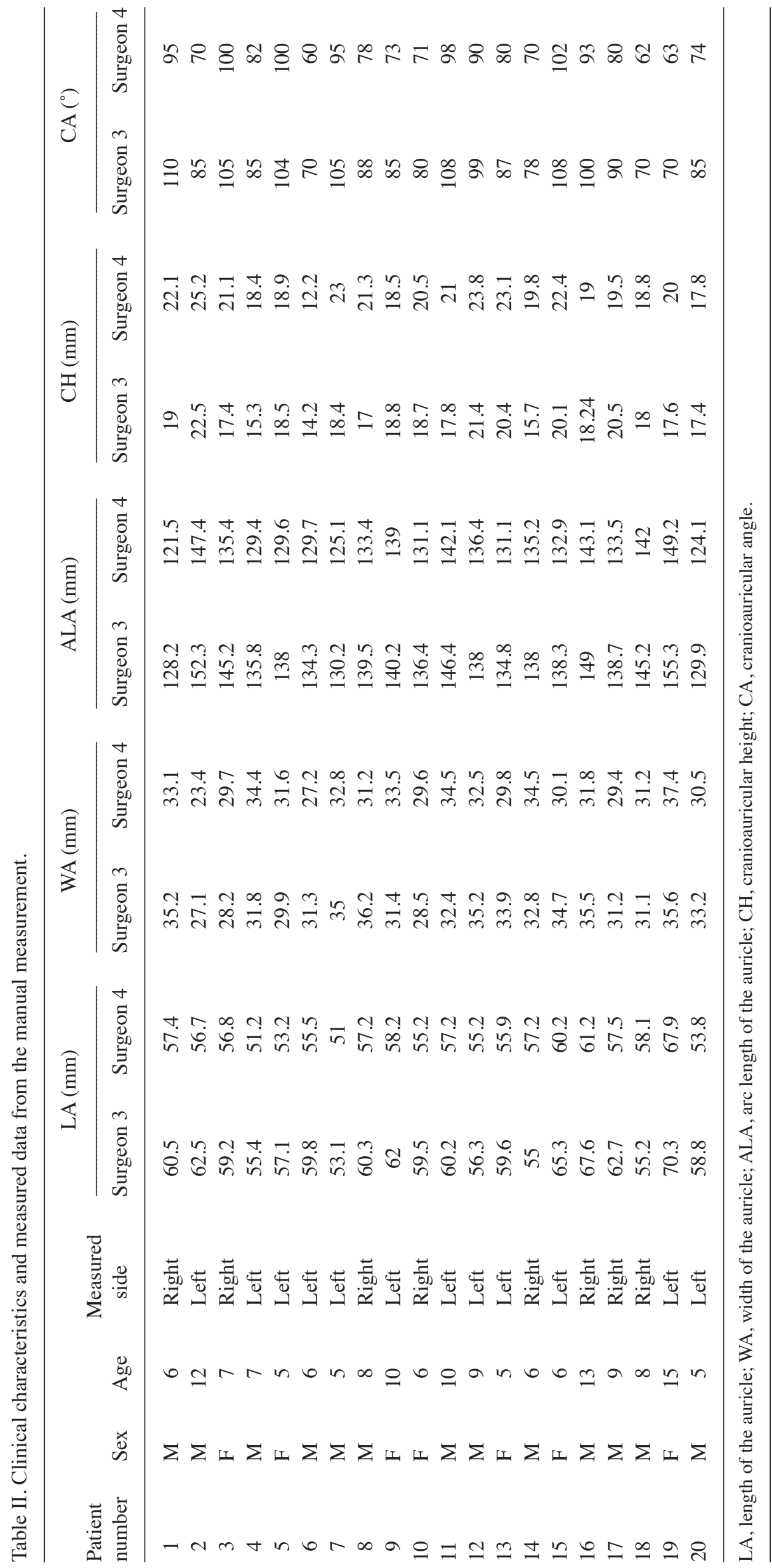


Table III. P-values and ICCs of the data obtained using the digital measurement method.

\begin{tabular}{lccccr}
$\begin{array}{l}\text { Statistical } \\
\text { item }\end{array}$ & LA & WA & ALA & CH & CA \\
\hline t value & -0.256 & -0.126 & 0.242 & -0.949 & -1.183 \\
P-value & 0.801 & 0.901 & 0.812 & 0.355 & 0.252 \\
ICC & 0.98 & 0.962 & 0.944 & 0.901 & 0.987 \\
\hline
\end{tabular}

Paired samples t-test was used for the comparison of data obtained by the two surgeons using digital measurement method. LA, length of the auricle; WA, width of the auricle; ALA, arc length of the auricle; $\mathrm{CH}$, cranioauricular height; $\mathrm{CA}$, cranioauricular angle; ICC, intra-class correlation coefficient.

method is inconvenient and time-consuming (7,9). In addition, another essential factor that influences the outcome of manual measurement is that contact with the ears results in ear distortion and introduces further variation to the data. To the best of our knowledge, this is the first study that attempted to unify the indices for the measurement of auricles using a highly precise and reliable digital method.

CT scans are widely used to collect data for $3 \mathrm{D}$ reconstruction $(10,17)$. It has been previously reported that exposure to radiation is a risk factor for the development of malignant tumors (18). Since clinical benefits should be considered for patients, especially for children, an alternative for CT scans would be beneficial. In the present study, a 3D surface scanner digitizing the intricate details of the auricle was used. A previous study has demonstrated minimal differences in the screenshots of specimen surfaces obtained using a CT scan and an Artec Space Spider scanner (19). Blue light 3D scanning technology used in the Artec Spider does not emit radiation during the scanning process and is therefore harmless to patients (20).

Digitizing software is frequently applied in 3D reconstruction and measurement, and other aspects in the field of surgery $(21,22)$. In the present study, Mimics software was used for the measurement of straight-line distance, arc length and angle with an accuracy of $0.01 \mathrm{~mm}$ and $0.01^{\circ}$, which was markedly higher compared with previous reports $(6,9)$. Mimics software is an image processing software that can create 3D models, measurements and analysis of 3D images (23), but was mostly applied in oral and maxillofacial surgery $(10,22)$. Manual measurements and digital measurement has been compared previously. Some previous reports have drawn the conclusion that manual and digital measurements are similar and that they were both reliable $(24,25)$, while in another study the authors deemed the digital approach more reliable (26).

As the auricle can become easily deformed during manual measurement, error derived from distortion is common. The 3D scanning requires no ear contact, and therefore, introduces no distortion to the auricle, suggesting that digital measurement may be more accurate compared with manual measurement. The position of the measured subject and the perspective of the observer may also influence the measure-
Table IV. P-value and ICCs of the data obtained using the manual measurement method.

\begin{tabular}{lccccc}
$\begin{array}{l}\text { Statistical } \\
\text { item }\end{array}$ & LA & WA & ALA & CH & CA \\
\hline Z-value & -3.585 & -1.999 & N/A & N/A & N/A \\
t value & n/a & n/a & 11.188 & -4.845 & 12.471 \\
P-value & $<0.01$ & $<0.01$ & $<0.01$ & $<0.01$ & $<0.01$ \\
ICC & 0.630 & 0.526 & 0.790 & 0.546 & 0.807
\end{tabular}

Wilcoxon signed-rank test or paired samples t-test was used for the comparison of data obtained by the two surgeons using manual measurement method, as appropriate. LA, length of the auricle; WA, width of the auricle; ALA, arc length of the auricle; $\mathrm{CH}$, cranioauricular height; $\mathrm{CA}$, cranioauricular angle; ICC, intra-class correlation coefficient; N/A, not available.

ments, as previously described (27). Following data collection and processing, all operations were performed in silico, where the 3D images could be scrutinized at will to expose details if necessary, especially for the selection of measurement points of the auricle. Therefore, combined with traditional tools, this method can provide a highly precise auricular measurements without contact-mediated distortion.

Results from the present demonstrated the digital measurement method is replicable and reliable, which carries advantages over the manual method. No significant differences were found between measurements of the five auricular parameters made by the two surgeons using the digital method. However, significant differences were found between measurements made by the two surgeons using the manual method with non-uniformed indices. An important indicator for the assessment of a new measurement method is the reproducibility (28). In the present study, ICC using a scale of $0-1$ was calculated for this purpose. The ICC values calculated from digital measurements ranged from 0.901 to 0.987 demonstrating excellent reproducibility by independent investigators. In comparison, ICC values from manual measurements ranged from 0.526 to 0.807 . This finding suggests that the standardized digital measurement method is more repeatable and reliable compared with the traditional manual method. Subsequently, Pearson's product moment correlation analysis was utilized to assess the correlation between $\mathrm{CH}$ and $\mathrm{CA}$ in addition to the correlation between LA and WA. A moderate but significant positive correlation was observed between $\mathrm{CH}$ and $\mathrm{CA}$ $(0.4<\mathrm{r}<0.7)$, suggesting that higher ear location is correlated with a larger CA. In the process of costal cartilage carving, increasing base thickness would increase the cranioauricular angle. However, no positive correlation between LA and WA was found $(r=0.206)$.

A comprehensive auricular measurement method should specifically analyze the main characteristics of the ear shape. The significance of the LA and WA, which are used widely, is well known (5). Some diagnosis of ear deformations such as the prominent ear is dependent on cranioauricular angles (7). In addition, $\mathrm{CH}$ and $\mathrm{CA}$ are the key indices in diagnosing microtia (20). The ALA is becoming a very promising index in 
the diagnosis of constricted ear and other abnormalities which are associated with substantial helix changes (29). Therefore, in the present study, a total of five parameters were measured, which contain almost all the necessary data for the diagnosis, pre-operation design and outcome evaluation of a variety of auricular abnormalities.

This study has a number of limitations. No matter which measurement method is used, inconsistent measurement points can occur on different ears, resulting in the value measured to not be representative of the maximum value. For instance, in the measurement of the WA, many investigators choose the intersection of the helix and the skull surface as the origin, but these intersections can vary markedly in different cases (5). However, in the evaluation of therapy outcomes, it is paramount that the same measurement points are chosen before and after therapy for precise measurements to be achieved.

In conclusion, measurement of LA, WA, ALA, CH and CA using the standardized digital method combining 3D scanning technology and Mimics software is more precise, convenient, reproducible and reliable compared with the traditional manual method. The LA, WA, ALA, CH and CA measured for the evaluation of different auricle abnormalities and healthy auricles are comprehensive, efficient and simple, and a positive correlation between $\mathrm{CH}$ and $\mathrm{CA}$ was found in the present study.

\section{Acknowledgements}

Not applicable.

\section{Funding}

The research reported in this publication was supported by the CAMS Innovation Fund for Medical Sciences (grant nos. CAMS-I2M-1-007 and CIFMS 2016-12M-2-006), PUMC Graduate Innovation Fund (grant no. 2018-1002-02-20), the Beijing Municipal Science \& Technology Commission (grant no. Z161100000516098) and PUMC Youth Fund and the Fundamental Research Funds for the Central Universities (grant no. 33320140171).

\section{Availability of data and materials}

All data generated or analyzed during this study are included in this published article.

\section{Authors' contributions}

DW, HYJ, BP and LL designed the current study. DW, HYS and XBY contributed to acquisition and analysis of patient data. LL, HYJ, QHY and LRH supervised and managed the study. HYJ, QHY and LRH performed the surgeries. DW and LL wrote the manuscript. HYJ, QHY, LRH, HYS, XBY and BP revised the manuscript. DW, HYJ and LL contributed to the acquisition of funding. All authors gave their approval to the final version of this manuscript.

\section{Ethics approval and consent to participate}

Ethical approval of the study was obtained from the Institutional Review Board of Plastic Surgery Hospital of
Peking Union Medical College. All parents or guardians of the patients granted informed consent to participate in the present study.

\section{Patient consent for publication}

Informed consents from all patients included in the current study were obtained for publication.

\section{Competing interests}

The authors declare that they have no competing interests.

\section{References}

1. Pawar SS, Koch CA and Murakami C: Treatment of prominent ears and otoplasty: A contemporary review. JAMA Facial Plast Surg 17: 449-454, 2015.

2. Mohammadi AA, Imani MT, Kardeh S, Karami MM and Kherad M: Non-surgical management of congenital auricular deformities. World J Plast Surg 5: 139-147, 2016.

3. Akter F, Mennie JC, Stewart K and Bulstrode N: Patient-reported outcome measures in microtia surgery. J Plast Reconstr Aesthet Surg 70: 416-424, 2017.

4. Farkas LG: Anthropometry of the normal and defective ear. Clin Plast Surg 17: 213-221, 1990.

5. Zhao H, Lin G, Seong YH, Shi J, Xu J and Huang W: Anthropometric research of congenital auricular deformities for newborns. J Matern Fetal Neonatal Med 32: 1176-1183, 2019.

6. Leclère FM, Trelles M and Mordon SR: Cartilage reshaping for protruding ears: A prospective long term follow-up of 32 procedures. Lasers Surg Med 43: 875-880, 2011.

7. da Silva Freitas R, Sanchez ME, Manzotti MS, Baras F, Ono MC and de Oliveira e Cruz GA: Comparing cephaloauricular and scaphaconchal angles in prominent ear patients and control subjects. Aesthetic Plast Surg 32: 620-623, 2008.

8. Kobayashi S and Maegawa J. Ear elevation using 2-tiered costal cartilage on the same side as the reconstructed framework. J Craniofac Surg 22: 1796-1799, 2011.

9. Jiafeng L, Jiaming S and Xiaodan L: Auricular reconstruction using a novel three-flap technique improves the auriculocephalic angle. J Plast Reconstr Aesthet Surg 69: 1430-1435, 2016.

10. You W, Liu LJ, Chen HX, Xiong JY, Wang DM, Huang JH, Ding JL and Wang DP: Application of 3D printing technology on the treatment of complex proximal humeral fractures (Neer3-part and 4-part) in old people. Orthop Traumatol Surg Res 102: 897-903, 2016.

11. Zhao X, Yu RT, Li JS, Xu K and Li X: Clinical value of multi-slice 3-dimensional computed tomographic angiography in the preoperative assessment of meningioma. Exp Ther Med 6: 475-478, 2013.

12. Glittenberg $C$ and Binder S: Using $3 D$ computer simulations to enhance ophthalmic training. Ophthalmic Physiol Opt 26: 40-49, 2006.

13. Li L, Zeng L, Lin ZJ, Cazzell M and Liu H: Tutorial on use of intraclass correlation coefficients for assessing intertest reliability and its application in functional near-infrared spectroscopy-based brain imaging. J Biomed Opt 20: 50801, 2015.

14. Thorne $\mathrm{CH}$ and Wilkes G: Ear deformities, otoplasty, and ear reconstruction. Plast Reconstr Surg 129: e701-e716, 2012.

15. Lee BM, Kang SJ and Sun H: Simple aesthetic correction for patients with acute auriculocephalic angle. Arch Craniofac Surg 16: 24-28, 2015.

16. Jiang H, Pan B, Zhao Y, Lin L, Liu L and Zhuang H: A 2-stage ear reconstruction for microtia. Arch Facial Plast Surg 13: 162-166, 2011.

17. Shinohara H, Matsuo K, Hataya Y and Taki K: Correlation between projection of the ear, the inferior crus, and the antihelical body: Analysis based on computed tomography. Scand J Plast Reconstr Surg Hand Surg 41: 288-292, 2007.

18. Johns AL, Lucash RE, Im DD and Lewin SL: Pre and post-operative psychological functioning in younger and older children with microtia. J Plast Reconstr Aesthet Surg 68: 492-497, 2015.

19. DellaCroce FJ, Green S and Aguilar EF III: Framework growth after reconstruction for microtia: Is it real and what are the implications? Plast Reconstr Surg 108: 1479-1486, 2001. 
20. Zhou J, Pan B, Yang Q, Zhao Y, He L, Lin L, Sun H, Song Y, Yu X, Sun $Z$ and Jiang H: Three-dimensional autologous cartilage framework fabrication assisted by new additive manufactured ear-shaped templates for microtia reconstruction. J Plast Reconstr Aesthet Surg 69: 1436-1444, 2016.

21. Khamaisy S, Zuiderbaan HA, Thein R, Nawabi DH, Joskowicz L and Pearle AD: Coronal tibiofemoral subluxation: A new measurement method. Knee 21: 1069-1071, 2014.

22. Huang JW, Shan XF, Lu XG and Cai ZG: Preliminary clinic study on computer assisted mandibular reconstruction: The positive role of surgical navigation technique. Maxillofac Plast Reconstr Surg 37: 20, 2015.

23. Asif MK, Nambiar P, Mani SA, Ibrahim NB, Khan IM and Sukumaran P: Dental age estimation employing CBCT scans enhanced with Mimics software: Comparison of two different approaches using pulp/tooth volumetric analysis. J Forensic Leg Med 54: 53-61, 2018.

24. Leifert MF, Leifert MM, Efstratiadis SS and Cangialosi TJ: Comparison of space analysis evaluations with digital models and plaster dental casts. Am J Orthod Dentofacial Orthop 136 16.e1-e4; discussion 16, 2009.

25. Bootvong K, Liu Z, McGrath C, Hägg U, Wong RW, Bendeus M and Yeung S: Virtual model analysis as an alternative approach to plaster model analysis: Reliability and validity. Eur J Orthod 32: 589-595, 2010
26. Santoro M, Galkin S, Teredesai M, Nicolay OF and Cangialosi TJ: Comparison of measurements made on digital and plaster models. Am J Orthod Dentofacial Orthop 124: 101-105, 2003.

27. Correia GD, Habib FA and Vogel CJ: Tooth-size discrepancy: A comparison between manual and digital methods. Dental Press J Orthod 19: 107-113, 2014.

28. Nawi N, Mohamed AM, Marizan Nor M and Ashar NA Correlation and agreement of a digital and conventional method to measure arch parameters. J Orofac Orthop 79: 19-27, 2018.

29. Wang D, Pan B, Lin L, Yang Q, He L, Song Y, Zhou J and Jiang H: New methods for specialized subjective and high-precision objective evaluation of constricted ears: A pilot study. Medicine (Baltimore) 97: e12997, 2018

(i) () $(9$ This work is licensed under a Creative Common EY NO ND Attribution-NonCommercial-NoDerivatives 4.0 International (CC BY-NC-ND 4.0) License. 